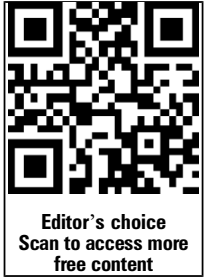

- Additional material is published online only. To view please visit the journal online (http://dx.doi.org/10.1136/ gutjnl-2014-307240)

${ }^{1}$ Department of Veterinary Biosciences, Microbiology, University of Helsinki, Helsinki, Finland

${ }^{2}$ Immune Biology Research Program, Department of Bacteriology and Immunology, University of Helsinki, Helsinki, Finland

${ }^{3}$ NIHR Biomedical Research Unit, Nottingham Digestive Diseases Centre, University Hospital, Nottingham, UK ${ }^{4}$ Sir Peter Mansfield Magnetic Resonance Centre, University of Nottingham, Nottingham, UK ${ }^{5}$ Laboratory of Microbiology, Wageningen University, Wageningen, The Netherlands

\section{Correspondence to}

Jonna Jalanka, Department of Veterinary Biosciences, University of Helsinki P.O. Box 66, Helsinki Fl-00014, Finland Jonna.Jalanka@Helsinki.fi

Received 14 March 2014 Revised 28 November 2014 Accepted 29 November 2014 Published Online First 19 December 2014

\section{SLinked}

http://dx.doi.org/10.1136/ gutjnl-2014-308937

\section{CrossMark}

To cite: Jalanka J, Salonen A, Salojärvi J, et al. Gut 2015;64:1562-1568.

\title{
Effects of bowel cleansing on the intestinal microbiota
}

\author{
Jonna Jalanka, ${ }^{1}$ Anne Salonen, ${ }^{2}$ Jarkko Salojärvi, ${ }^{1}$ Jarmo Ritari, ${ }^{1}$ Outi Immonen, ${ }^{1}$ \\ Luca Marciani, ${ }^{3}$ Penny Gowland, ${ }^{4}$ Caroline Hoad ${ }^{4}$ Klara Garsed, ${ }^{3}$ Ching Lam, ${ }^{3}$ \\ Airi Palva, ${ }^{1}$ Robin C Spiller, ${ }^{3}$ Willem M de Vos ${ }^{1,2,5}$
}

\section{ABSTRACT \\ Objective An adequate bowel cleansing is essential for \\ Significance of this study} a successful colonoscopy. Although purgative consumption is safe for the patient, there is little consensus on how the intestinal microbiota is affected by the procedure, especially regarding the potential long-term consequences.

Design 23 healthy subjects were randomised into two study groups consuming a bowel preparation (Moviprep), either in two separate doses of $1 \mathrm{~L}$ or as a single 2-L dose. Participants donated faecal samples at the baseline, after bowel cleansing, 14 and 28 days after the treatment. The intestinal microbiota composition was determined with phylogenetic microarray as well as quantitative $P C R$ analysis and correlated with the previously quantified faecal serine proteases.

Results The lavage introduced an instant and substantial change to the intestinal microbiota. The total microbial load was decreased by 31 -fold and $22 \%$ of the participants lost the subject-specificity of their microbiota. While the bacterial levels and community composition were essentially restored within 14 days, the rate of recovery was dose dependent: consumption of the purgative in a single dose had a more severe effect on the microbiota composition than that of a double dose, and notably increased the levels of Proteobacteria, Fusobacteria and bacteria related to Dorea formicigenerans. The abundance of the latter also correlated with the amount of faecal serine proteases that were increased after purging.

Conclusions Our results suggest that the bowel cleansing using two separate dosages introduces fewer alterations to the intestinal microbiota than a single dose and hence may be preferred in clinical practice.

\section{INTRODUCTION}

Adequate bowel cleansing is essential for successful endoscopic examinations as well as colonic surgeries. The efficacy of the bowel cleansing products and their safety to the patient have been studied extensively ${ }^{1}$ however, the effects of lavage on the colonic microbiota have so far been poorly characterised. Several purgatives are used in the clinical practice; one widely used is the polyethylene glycol (PEG) supplemented with electrolytes. It introduces an osmotic flow of fluids to the GI tract, drastically altering the normal colonic environment. The ingested PEG electrolyte solution and the increased volume of fluids flushes out the luminal bacteria, introduces oxygen into the normally anaerobic colonic ecosystem ${ }^{2}$ and reduces the nutrition supply
What is already known on this subject?

- Changes in the intestinal microbiota following bowel cleansing have been detected. However, a detailed description or the long-term effects to the microbiota has not been characterised.

- Two different dosing methods are used in the clinical practice. The effect of the dosing to the intestinal microbiota has not been addressed.

- Faecal serine proteases are predominantly of pancreatic origin and shown to be increased in IBS patients with diarrhoea and after bowel cleansing.

\section{What are the new findings?}

- The majority of the intestinal microbiota recovered to the baseline composition after bowel preparation.

- Subjects consuming a single dose $(2 \mathrm{~L})$ of the purgative showed increased abundance of several taxa including Proteobacteria and bacteria related to Dorea formicigenerans in the follow-up samples and less efficient microbial recovery than the subjects given two separate $1 \mathrm{~L}$ dosages.

How might it impact on clinical practice in the foreseeable future?

- These data provide insight into the microbiota changes associated with purgative lavage and may help in the understanding of the changes seen in IBS and other diarrhoeal diseases.

- Two separate dosages of purgative introduced fewer alterations to the intestinal microbiota and resulted in a colon with a lower bacterial load than the single dose.

for the intestinal bacteria. All these rapid changes may affect the microbial ecosystem.

Maintaining homeostasis between the intestinal microbiota and the host is a key element for their mutualistic relationship. In general, the intestinal microbiota of healthy adults is characterised by high subject-specificity and stability over several years. ${ }^{3-5}$ Disturbances such as antibiotic treatments are known to have initially a profound effect, but the overall microbiota tends to recover remarkably fast to resemble its original composition. ${ }^{6}$ Some of the bacterial taxa suffer from such disturbances more than others and for instance certain Bacteroides spp have been shown to be strongly reduced after antibiotic 
treatment. ${ }^{6}$ The occurrence of such drastic changes may give pathobionts, normally suppressed by the commensal microbiota, a chance to proliferate. ${ }^{7}$ Moreover, these alterations resemble the tipping point changes that may affect the resilience of the microbial ecosystem as we recently reported. ${ }^{8}$

Previous studies focusing on the effects of bowel cleansing on the intestinal microbiota have not been able to detect consistent changes. $^{9-12}$ Several reports with limited number of subjects have indicated that lavage does not alter the microbial diversity even when the total bacterial load was halved. ${ }^{10-12}$ It has been reported that bowel cleansing might cause temporary changes in the mucus layer ${ }^{13}$ and microbial changes in the mucosal tissue after bowel preparation has shown a trend towards increased amounts of Gram-negative bacteria belonging to Proteobacteria. ${ }^{10}{ }^{12}$ However, in many cases, the observed microbial aberrations following the bowel cleansing were not coherent and found to be very subject-specific. Moreover, a detailed description on the microbial recovery has been lacking. The main reason for the inconclusive evidence for the impact of purgative agents on the intestinal microbiota can be ascribed to small numbers of participants, the inclusion of healthy subjects and patients, and the lack of analytical depth. In the present study, we increased the number of subjects and used a global and deep analysis method for determining the changes in the intestinal microbiota. A recent study showed that increased transit, due to diarrhoea or bowel cleansing, resulted in increased levels of faecal serine proteases (FSPs), which were thought to represent reduced bacterial degradation of endogenous pancreatic enzymes. ${ }^{14}$ This may be important because faecal proteases correlate with urgency in IBS with diarrhoea, a key feature of IBS developing after a bout of infectious gastroenteritis, which often begins with an episode of purging. These changes have now been correlated in this current study to microbial changes in the host. Moreover, there is debate about the merits of two different regimes of giving PEG electrolyte solution (single $2 \mathrm{~L}$ dose or two doses of $1 \mathrm{~L}$ separated by $12 \mathrm{~h}$, hereafter known as 'split dose'). We examined the immediate effects of the two doses on the intestinal microbiota as well as the impact on the microbial recovery rate. The results indicate that the split dose of the purgative produced less qualitative changes in the intestinal microbiota and a greater reduction in bacterial concentration.

\section{MATERIALS AND METHODS}

\section{Study subjects and design}

The study aimed to investigate the effects of consuming a bowel preparation Moviprep (100 g/L PEG 3350, sodium chloride $46 \mathrm{mmol} / \mathrm{L}$, sodium sulfate $53 \mathrm{mmol} / \mathrm{L}$ and ascorbate $30 \mathrm{mmol} / \mathrm{L}$ ) on the intestinal microbiota and to determine whether the two dosing regimens as used in the clinical practice introduces any longlasting effects in the recovery rate. ${ }^{15}$ In total, 23 healthy subjects were recruited from the staff and students of Nottingham University Hospitals and randomly assigned into groups receiving two different dosages of the study substance (table 1, detailed description of the patients in the online supplementary material). The study was approved by the National Research Ethics Service (approval 10/H0906/50), the NHS Trust R\&D (approval 10GA018) and the Medicines and Healthcare products Regulatory Agency (MHRA Clinical Trial Authorization CTA 03057/0045/ 001-0001, protocol 10050). All participants gave informed written consent. Group I received a split dose of 2 L of PEG electrolyte solution; the first litre in the evening and the second litre on the following morning. Group II consumed a single dose of $2 \mathrm{~L}$ of PEG electrolyte solution on the morning of the study. Four stool samples were collected from the participants. These included a baseline sample donated a day before the bowel cleansing, a sample
Table 1 Subject demographics, SEM in brackets (all, $p>0.05$ )

\begin{tabular}{lll}
\hline & Split dose & Single dose \\
\hline Sex & $5 \mathrm{M}, 6 \mathrm{~F}$ & $6 \mathrm{M}, 6 \mathrm{~F}$ \\
Age & $27(3)$ & $25(2)$ \\
Height $(\mathrm{m})$ & $170.6(3.3)$ & $171.7(2.6)$ \\
Weight $(\mathrm{Kg})$ & $67.1(3.3)$ & $68.9(2.8)$ \\
BMl $\left(\mathrm{kg} / \mathrm{m}^{2}\right)$ & $23.0(0.6)$ & $23.3(0.6)$ \\
\hline BMI, Body Mass Index. & &
\end{tabular}

immediately after the lavage and two follow-up samples 14 and 28 days after the bowel cleansing (figure 1). The participants were advised to collect the lavage sample on the study day, when the faecal material was liquid and free from solids.

\section{Analysis of the faecal microbiota composition}

Samples collected at home were immediately frozen in domestic freezers at $-20^{\circ} \mathrm{C}$ prior to the delivery to the Biomedical Research Centre within $2 \mathrm{~h}$ for storage at $-70^{\circ} \mathrm{C}$. Samples collected during the study day were immediately frozen at $-70^{\circ} \mathrm{C}$. The faecal microbial DNA was extracted by using a previously described and validated repeated bead-beating method. ${ }^{16}$ The faecal samples obtained immediately after lavage were very dilute. In order to concentrate the sample, the extraction protocol was preceded by an additional centrifugation of either 2 or $10 \mathrm{~mL}(4000 \mathrm{~g}, 10 \mathrm{~min}$, $+4^{\circ} \mathrm{C}$ ) of the original sample into a faecal pellet (mean weight $0.36 \mathrm{~g}, \mathrm{SD}=0.13 \mathrm{~g}$ ). The microbiota composition was analysed by using a previously validated and benchmarked custom made phylogenetic microarray (HITChip). ${ }^{3}{ }^{17} 18$ It targets two hypervariable regions (V1 and V6) of the 16S rRNA gene and covers over 1000 bacterial phylotypes detected in the human GI track. The raw signal intensities were normalised as described previously using by Robust Probabilistic Averaging method ${ }^{8} 19$ that takes into account the possible cross-hybridisation of the probes. The probe signal intensities were summarised into 130 genus-like taxonomic groups referred to with a species name and relatives according to the nearest cultured relative, for example, Escherichia coli et rel. The phylogenetic microarray was supplemented with quantitative PCR (qPCR) analysis of the total bacteria, ${ }^{20}$ methanogenic archaea ${ }^{21}$ and in a subset of samples the amount of human the $\beta$-actin gene was also measured in order to estimate the amount of host DNA ${ }^{22}$ (detailed in online supplementary table S1). The concentration step conducted on the faecal samples collected immediately after lavage was taken into account when analysing the $\mathrm{qPCR}$ results.

\section{Predicting the functional potential of the intestinal microbiota}

The FSPs were quantified from all donated samples. The results and the protocol have been published previously. ${ }^{14}$ In short, the

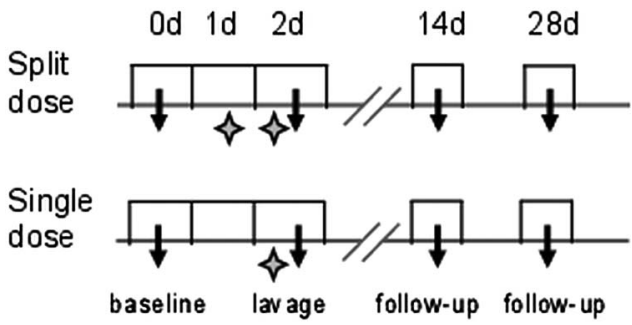

Figure 1 The study design. The consumption of the polyethylene glycol electrolyte solution is indicated with an asterisk and the faecal sample collection points are indicated with an arrow. 
FSP levels were determined by using a non-specific proteolysis of azo-casein and measuring the absorbance of the resultant-free azo-peptides in the supernatant. ${ }^{23}$

In this paper, we determine the correlation between the total bacterial load and FSP levels using Pearson's coefficient (r). The potential functions of the microbiota based on the 16S rRNA information were predicted using the newly developed PICRUSt software (V.1.0.0.0 running on Galaxy). ${ }^{24}$ The $16 \mathrm{~S}$ bacterial sequences targeted and quantified with HITChip were mapped to Greengenes reference OTUs (V.13_5 $)^{25}$ with BLAST. The QIIME $^{26}$ analysis pipeline including PICRUSt was applied. The method estimates the functional composition of the metagenome based on the available reference genomes, and results in predictions of KEGG-orthologue $(\mathrm{KO})^{27}$ abundances, which were used in the downstream analyses. KOs having total counts below 13000 were removed as background (detailed description in the online supplementary material).

\section{Statistical analysis}

All statistical analyses were carried out with 10-base logarithmtransformed data and performed with scripts in R, V.3.0.1. The similarity of the microbiota profiles was determined using hierarchical clustering and Pearson's correlation based distance measure. Validation of the hierarchical clustering was performed using multiscale bootstrap resampling, implemented in $\mathrm{R}$ package 'pvclust'. The changes in the individual bacterial taxa between the study groups and time points were assessed by a linear mixed model using first order autoregressive covariance model to estimate correlation between consecutive time points. The resulting $\mathrm{p}$ values were adjusted for multiple comparisons using the Benjamini-Hochberg false discovery rate (FDR) correction. Only FDR-corrected $\mathrm{p}$ values below 0.05 were considered significant. Associations between the microbiota and the amounts of FSPs were assessed with non-parametric Spearman correlation test, and the subsequent $\mathrm{p}$ values were corrected using the BenjaminiHochberg FDR. Diversity, the measure of richness and evenness of a bacterial population, was calculated by using Shannon diversity index.

\section{RESULTS \\ Consumption of PEG electrolyte solution temporarily alters the intestinal microbiota composition}

We aimed to comprehensively address the overall effect of Moviprep treatment on the intestinal microbiota as well as the differences of the two dosages of the purgative (single or split dose of 2 L of PEG electrolyte solution), widely used in the clinical practice. First, we determined the effect of bowel cleansing on the total bacterial load, based on the total $16 \mathrm{~S}$ rRNA signal estimated with qPCR, taking into account the concentration step conducted on the lavage samples when extracting the microbial DNA. We found that the number of bacteria in the samples collected immediately after bowel cleansing was in average 34.7 -fold $(p<0.001)$ lower than in the normal faecal samples at baseline (figure 2A). Similarly, the numbers of methanogenic archaea per gram of faeces were also significantly decreased $(20$-fold, $\mathrm{p}<0.001)$ (figure 2B). However, all these numbers were restored to the baseline levels after 14 and 28 days. Accordingly, the similarity of the microbiota profiles showed a significant decrease during the lavage treatment when compared with the baseline (Pearson's correlation $r=0.93$, $\mathrm{p}<0.05$, figure $2 \mathrm{C}$ ). The microbiota of the follow-up samples resembled that of the baseline, with correlations averaging 0.97 . Remarkably, the subjects in the single dose group had a higher bacterial load than subjects in the split dose group after the purgative treatment (25.3- and 64.7-fold changes, respectively, $p<0.05$, figure 2D). To verify the quality of the DNA extraction and GPCR results, we estimated the amount of human DNA in a random selection of subjects $(n=20)$ and found that on average there was 121000 -fold less human DNA than bacterial DNA in the faecal samples. Moreover, the ratio between the human and bacterial DNA did not differ between the baseline and lavage samples $(\mathrm{p}>0.1)$.

To study the microbiota changes in an individual level, we performed hierarchical clustering of the samples (figure $3 \mathrm{~A}$ ) and the obtained dendrogram was validated with extensive bootstrap analysis (see online supplementary figure S1). The intestinal microbiota composition is known to be subject-specific, which is also evident from this analysis that shows the grouping of repeated samples from the same individual. However, in five out of the 23 participants (22\%), lavage altered the microbiota composition so considerably that the subject-wise clustering was lost (figure 3A). Nevertheless, already 14 days after the bowel preparation the microbiota of these individuals had recovered to resemble its original form. Taken together, the majority of the microbiota returned to its original composition after the consumption of PEG electrolyte solution, although the lavage introduced a momentary substantial change.

\section{Specific changes in the microbiota at the time of purging}

The average microbiota composition in the baseline samples was in accordance with previous reports for healthy adults: ${ }^{28}$ a mean of $63 \%$ of Firmicutes, $24 \%$ of Bacteroidetes, $4 \%$ of Actinobacteria, 1\% of Verrucomicrobia and 1\% of Proteobacteria. There were no differences in the microbiota composition between the two study groups in this time point. Immediately after the lavage, the intestinal microbiota was significantly different than in the baseline samples, even on class or family level (figure 3B). There was a significant decrease in the genera belonging to Bacilli and genera affiliated with Clostridium cluster IV (table 2). More specifically, bacteria related to Ruminococcus bromii and Ruminococcus callidus were decreased 2.5- and 2.3-fold, respectively (all $\mathrm{p}<0.05$ ). The decrease of these bacteria was momentary, since in the 14-day follow-up samples all levels had returned to resemble the baseline, with the exception of $R$. bromii. In addition, we identified bacteria showing increased abundance at the time of lavage; these were predominantly members of the Proteobacteria phylum and Clostridium cluster IV. The largest differences compared with the baseline were detected in bacteria related to Dorea formicigenerans and Ruminococcus gnavus $(\mathrm{p}<0.05,2.1$ - and 2.7-fold changes, respectively). Notably, there was also approximately a twofold increase of several Proteobacteria, including Sutterella wadsworthia and Serratia $(\mathrm{p}<0.05)$, after lavage.

The lavage also affected the prevalence of the methanogenic archaea, as well as their abundance. It has been shown previously that the carriers of Methanobrevibacter smithii show stable levels of this commensal ${ }^{3}$ while only $45 \%-70 \%$ of people are carriers. ${ }^{16}$ In the present study, 13 out of the 23 participants $(57 \%)$ were carriers at the baseline (mean abundance in $\log 10$ scale was 8.74 per gram of faeces, $S D=0.90)$, two of whom did not have detectable levels of methanogens anymore after the lavage treatment. Both of these subjects received the split dose of the PEG electrolyte solution. On the other hand, there were five subjects who had detectable levels of methanogens only after the lavage. As the detection limit of the assay is as low as 100 copies per assay, the carrier status is unlikely to result from amplification that fluctuates below and above the detection limit, suggesting that the lavage indeed altered the prevalence of the intestinal methanogens. 
A

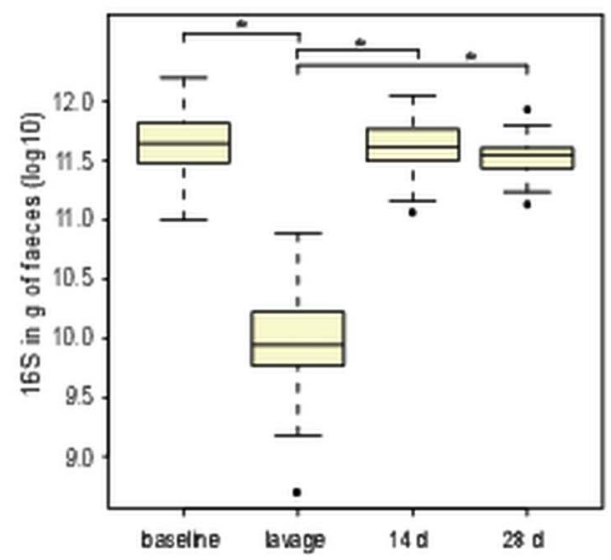

C

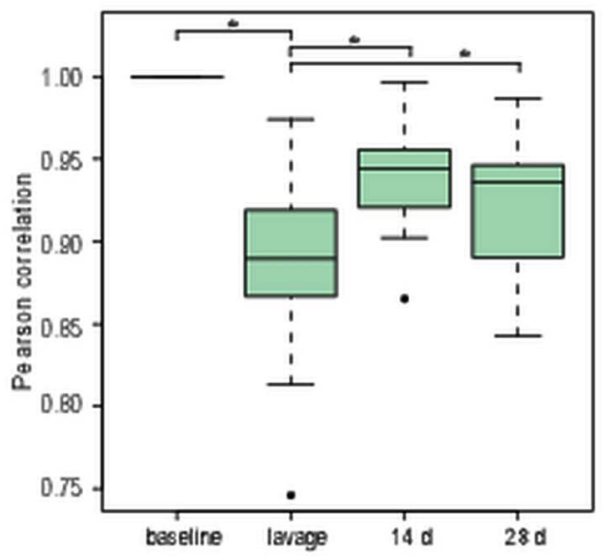

B

Methanogenic archaea

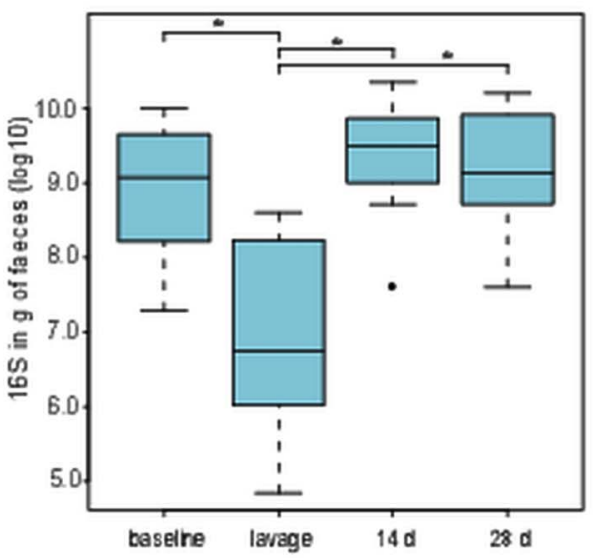

Total bacterial load per study group

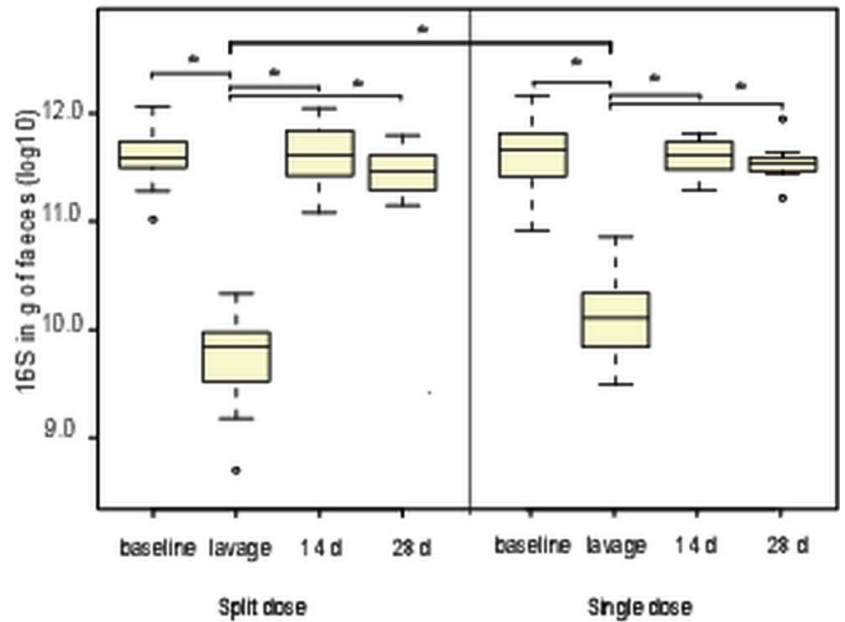

Figure 2 Effect of bowel cleansing for the (A) total bacteria, (B) methanogenic archaea, (C) the microbial recovery calculated as similarity of the microbial profile against the baseline sample and (D) differences in the total bacteria of the two dosage groups measured with quantitative PCR. Statistical significance is indicated with an asterisk.

While diversity has been shown to be decreased in diarrhoea diseases in general, ${ }^{29}$ we found no statistical differences in the diversity of the microbiota between the time points or treatment groups in agreement with prior studies addressing the impact of lavage. ${ }^{10-12}$ However, the ratio between Gram-positive and Gram-negative species changed drastically after the lavage from $5.3(\mathrm{SD}=4.8)$ at baseline to $9.2(\mathrm{SD}=7.5)$ at the first follow-up sample 14 days after the lavage $(p<0.05)$. The 28 -day follow-up sample showed a trend towards baseline levels (see online supplementary figure S2).

\section{Impact of PEG electrolyte solution dosage on the microbiota}

Although 2 weeks after purging the overall microbiota had returned to resemble the baseline, we aimed to study whether there would be differences in the bacterial recovery rate, and if these could be explained by the different dosing of the PEG electrolyte solution. We identified several bacterial groups including eight genera that did not return to their baseline abundance in the single dose group (table 3). The four genera belonging to Proteobacteria, predominantly bacteria related to Proteus, showed a significant increase still 28 days after purging. Overall, we detected a $19 \%$ increase of the reported bacteria (detailed in table 3) from the relative baseline levels of $4.4 \%$ $(\mathrm{SEM}=0.32 \%)$ to $5.4 \%(\mathrm{SEM}=0.24 \%)$ in the 28 -day follow-up samples. In the split dosing group, only bacteria related to Eggerthella lenta (belonging to Actinobacteria) differed significantly between the baseline and both 14- and 28-day follow-up samples $(1.4$-fold increase, $\mathrm{p}<0.05)$. In conclusion, the split dose of the PEG electrolyte solution has a less disturbing effect on the intestinal microbiota than the single dose. This was also shown by the better microbial recovery of Protebacteria after the lavage treatment with the split dose when compared with the single dose group (see online supplementary figure S3).

\section{Associations between the microbiota and FSPs}

It has been shown previously from this cohort that there was a significant fourfold temporarily increase of FSP of pancreatic origin immediately after lavage, possibly due to decreased bacterial degradation of pancreatic trypsin (see online supplementary figure S4A). ${ }^{14}$ To complement these findings, we addressed whether the total bacterial load and specific bacteria could be linked to the increase of FSP by correlating the bacterial and FSP abundances across the samples. Remarkably, the total bacterial load was found to be inversely associated with the FSP amounts $\quad(\mathrm{r}=-0.61, \mathrm{p}<0.05$, see online supplementary 

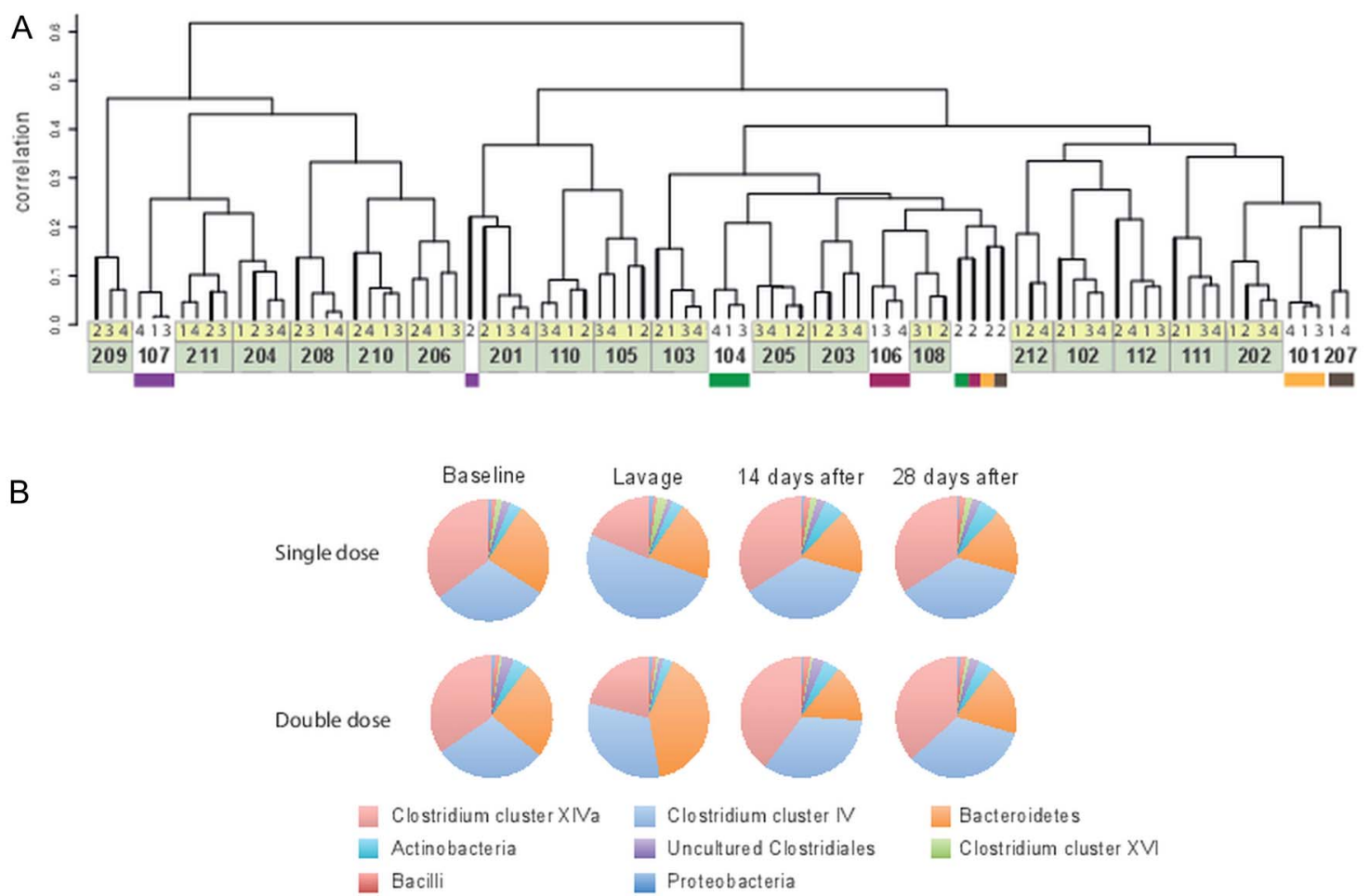

Figure 3 Effect of bowel cleansing on the microbial composition. (A) Hierarchical clustering of the microbiota profiles. Samples presenting subject-wise clustering are boxed, and the sampling time point is indicated with numbers 1=baseline, 2=lavage, 3=14-day follow-up and 4=28-day follow-up. The subjects not showing subject-wise clustering are indicated with a coloured line. The branch of the clustering tree corresponding to the samples collected immediately after lavage is indicated with a bold line. Missing samples: 10828 days, 207 baseline, 209 baseline and 212 14 days. (B) Microbiota composition, shown on the phylum level except for Firmicutes down to Clostridium clusters and Bacilli. The groups contributing more than $1 \%$ to the total microbiota are shown.

figure S4B). Moreover, we identified several bacterial genera that correlated significantly with the FSP amounts. The majority of the correlating taxa were from Clostridium cluster IV and XIVa (see online supplementary table S2), including bacteria related to Faecalibacterium prausnitzii $(\mathrm{r}=-0.4, \mathrm{p}<0.05)$, which decreased non-significantly during the lavage. A positive correlation was noted between the abundance of bacteria related to D. formicigenerans and FSP $(r=0.45, \mathrm{p}<0.05)$ (see online supplementary figure S5). By using PICRUSt, a computational method estimating the metagenomic content from the $16 \mathrm{~S}$ rRNA information, ${ }^{24}$ we investigated the relation between the FSPs and the predicted microbiota-derived functions related to protease activity. No indication for associations with serine protease-producing bacteria were found, in line with the previous observation that the FSP are provided by the host. ${ }^{14}$ In contrast, the predicted abundance of the serpin gene, encoding the bacterial serine proteinase inhibitor, was increased 1.42-fold after the lavage compared with the baseline and the 14-day follow-up sample $(\mathrm{p}<0.05)$.

\section{DISCUSSION}

Bowel cleansing is considered safe for healthy individuals. However, conflicting data have been reported on the impact of the purging treatment on the colonic microbiota, an important contributor to human health. Based on the present analysis, where the microbiota of 23 healthy subjects was investigated during and after routine Moviprep purging treatment, we conclude that the intestinal microbiota remains largely unaffected and returns to resemble the baseline after 2 weeks. The intestinal microbiota has been shown to retain its composition even after long periods of time $\mathrm{e}^{5}$ and samples from a single individual are considered to be highly subject-specific. ${ }^{4}$ Even though the intestinal microbiota composition was momentarily affected by the lavage treatment, it recovered from this disturbance rapidly. Immediately after the lavage, the amount and composition of the microbiota altered drastically so that in a subset of the study subjects (five out of 23) even the individuality of the microbiota was lost. However, the unique composition and the total bacterial load returned to the baseline levels already after 14 days.

Consumption of PEG increases the amount of water in the intestinal tract, washing out the luminal content and causing a substantial reduction of the faecal material, including the intestinal bacteria. Moreover, the rapid increase in bowel movements will flush out bacteria incapable of adhering to the gut mucosa, distorting the faecal bacterial composition compared with the normal state. Here we showed that the bowel cleansing treatment decreased the abundance of the members of Clostridium cluster IV and increased the members of Clostridium cluster XIVa and Proteobacteria (table 2). A recent study also showed that the bowel cleansing affects the quality and production of the protective mucus layer in the colon. ${ }^{13}$ Altered microbiota has been linked with diarrhoea in general. A large study addressing the intestinal microbiota in young children from low-income countries revealed an increase in Proteobacteria associated with moderate to severe diarrhoea ${ }^{29}$ suggesting that our findings may also provide insight into microbiota alterations due to osmotic diarrhoea.

It has been shown that the best result for bowel cleansing is achieved by using a split dose of the PEG electrolyte solution. ${ }^{30}$ The microbiota results presented here support the notion that the split dose is also superior for other reasons. The single dose of the PEG electrolyte solution caused a prolonged qualitative alteration to the microbiota. Furthermore, the bacterial load after cleansing was shown to be significantly higher than what was observed with the split dose. The microbiota changes included an 
Table 2 Statistically significant alterations in the microbiota of all participants between the baseline and lavage, shown as microarray signal intensity

\begin{tabular}{|c|c|c|c|}
\hline $\begin{array}{l}\text { Phylum/Firmicute } \\
\text { order }\end{array}$ & Genus-like group & $\begin{array}{l}\text { Baseline } \\
\text { (SEM) }\end{array}$ & $\begin{array}{l}\text { Lavage } \\
\text { (SEM) }\end{array}$ \\
\hline \multirow[t]{3}{*}{ Bacilli } & Gemella & $39(1)$ & $58(5)$ \\
\hline & $\begin{array}{l}\text { Lactobacillus plantarum } \\
\text { et rel. }\end{array}$ & $811(30)$ & $743(17)$ \\
\hline & Weissella et rel. & $62(4)$ & $50(2)$ \\
\hline \multirow[t]{4}{*}{ Clostridium cluster IV } & Clostridium cellulosi et rel. & 13375 (3347) & $6674(1264)$ \\
\hline & Clostridium leptum et rel. & 7408 (1114) & $4185(686)$ \\
\hline & $\begin{array}{l}\text { Ruminococcus bromii } \\
\text { et rel. }\end{array}$ & 8039 (1990) & $3281(797)$ \\
\hline & $\begin{array}{l}\text { Ruminococcus callidus } \\
\text { et rel. }\end{array}$ & 6959 (1203) & $3009(585)$ \\
\hline Clostridium cluster IX & Veillonella & $252(27)$ & $431(44)$ \\
\hline \multirow[t]{2}{*}{$\begin{array}{l}\text { Clostridium cluster } \\
\text { XIVa }\end{array}$} & $\begin{array}{l}\text { Dorea formicigenerans } \\
\text { et rel. }\end{array}$ & 15697 (1743) & 32398 (4187) \\
\hline & $\begin{array}{l}\text { Ruminococcus gnavus } \\
\text { et rel. }\end{array}$ & $3965(630)$ & $10652(2170)$ \\
\hline \multirow[t]{4}{*}{ Proteobacteria } & Aquabacterium & $59(6)$ & $128(22)$ \\
\hline & Haemophilus & $103(12)$ & $153(19)$ \\
\hline & Serratia & $85(13)$ & $182(48)$ \\
\hline & $\begin{array}{l}\text { Sutterella wadsworthia } \\
\text { et rel. }\end{array}$ & $1820(790)$ & $3888(931)$ \\
\hline
\end{tabular}

et rel., and relatives.

increase of several facultative aerobes such as Fusobacteria, Proteobacteria and D. formicigenerans. Interestingly, this reflects a pattern seen in some disease states such as IBD and inflammation. $^{31-33}$ It is known that purging leads to the introduction of oxygen into the normally anaerobic colonic ecosystem. ${ }^{2}$ This could explain the increased amounts of Proteobacteria, as these are capable of respiration. Moreover, bacteria related to D. formicigenerans were increased almost twofold after the single treatment of purgative. A similar increase of these mucosalocated bacteria has been described in IBS, ${ }^{34} \mathrm{UC}^{31}$ as well as in non-alcoholic fatty liver disease. ${ }^{32}$ While we have no evidence that the observed changes in the bacterial taxa may have a health impact in the healthy subjects analysed here, it cannot be excluded that the increase of the implicated organism have adverse health effects. This underlines the importance of investigation of microbiota changes after lavage in healthy subjects before extrapolating the results into different patient groups.

Elevated FSP levels have been described in IBS patients and are considered to increase the intestinal permeability and potentially lead to visceral hypersensitivity. ${ }^{35}$ In this study, the lavage introduced a momentary increase in the intestinal of FSP and $\mathrm{pH}$ levels at the point of lavage. ${ }^{14}$ The increase in $\mathrm{pH}$ might reflect the loss of short-chained fatty acids and could allow the Proteobacteria to flourish. We have previously shown that increased FSP correlates with urgency ${ }^{14}$ and when transit is slowed by means of a $5 \mathrm{HT} 3$ receptor antagonist, Ondansetron, the decline in FSP correlates with the increase in transit, suggesting FSP is an important factor in IBS-D. Previous studies have also shown that the majority of the FSP are of host origin. ${ }^{14}$ Normal pancreas secretes approximately $500 \mathrm{mg}$ of protease daily but faecal excretion is less than $5 \mathrm{mg} .{ }^{36}$ Rat studies using broad-spectrum antibiotics suggest that this decrease is largely due to bacterial degradation. ${ }^{37}$ We showed a negative association between the decrease in the total bacterial load and the levels of FSP. This suggests that having normal amounts of intestinal bacteria ensures an adequate degradation of endogenous pancreatic protease thus avoiding sensitisation of the rectum. The observation that the increased FSP levels are associated with different microbial composition is in line with earlier studies in IBS patients. ${ }^{38}$ To further understand the meaning of the associations between the FSP and microbiota, we applied the newly developed software PICRUSt ${ }^{24}$ that used the abundance of bacterial taxa and compared them with sequenced reference genomes. Immediately after lavage, the levels of serpin genes were elevated, implying that the microbiota responded to the increased FSP amounts. It is tempting to speculate that this may explain the increase of bacteria related to $D$. formicigenerans immediately after the lavage, since inspection of the genome of Dorea spp revealed the presence of a serpin gene (accession number for the protein WP_022318855). This suggests that bacteria such as that related to $D$. formicigenerans could cope with the increased amount of the FSP immediately after the lavage. Furthermore, these bacterial serpins might reduce the effect of FSP on gut physiology. However, our present analysis does not allow for differentiating whether the observed changes in the microbiota composition were due to flushing out or a decreased proliferation. We showed that increased abundance of the indicated species and infer that these organisms have been multiplying as a result of the changes induced by the purgative treatment. These could be an increased level of oxygen as well as increased levels of FSP. Whether this associates with any physiological effect on the host or is just an adaptation of the microbiota to changed conditions such as increased levels of FSP or luminal oxygen warrants further studies.

Overall, our results indicate that bowel cleansing has a limited effect on the subjects' intestinal microbiota. We show that the

Table 3 Significant microbial changes between baseline and the follow-up samples in the single dose group shown as microarray signal intensity

\begin{tabular}{|c|c|c|c|c|}
\hline Phylum/Firmicute order & Genus-like group & Baseline (SEM) & 14d (SEM) & 28d (SEM) \\
\hline Clostridium cluster IV & Clostridium orbiscindens et rel. & 11971 (1773) & 18011 * (1581) & $20269 *(3452)$ \\
\hline Clostridium cluster XIVa & Dorea formicigenerans et rel. & 14628 (3295) & $18902 *(3164)$ & $21854^{*}(4603)$ \\
\hline Clostridium cluster XVIII & Clostridium ramosum et rel. & $776(66)$ & $533 *(152)$ & $635(305)$ \\
\hline Fusobacteria & Fusobacteria & $354(10)$ & $372(4)$ & $379^{*}(7)$ \\
\hline \multirow[t]{4}{*}{ Proteobacteria } & Megamonas hypermegale et rel. & $39(1)$ & $42(3)$ & $44^{*}(2)$ \\
\hline & Moraxellaceae & $78(1)$ & $95(13)$ & $90 *(7)$ \\
\hline & Novosphingobium & $47(1)$ & $54(2)$ & $55^{*}(3)$ \\
\hline & Proteus et rel. & $313(7)$ & $341 *(27)$ & $341 *(21)$ \\
\hline
\end{tabular}

*Statistically significantly different from baseline, $\mathrm{p}<0.05$.

et rel., and relatives. 
microbiota recovers to resemble the baseline composition when the PEG electrolyte solution was consumed in two $1 \mathrm{~L}$ doses. However, when only the single $2 \mathrm{~L}$ dose was consumed, the microbiota differed from the baseline sample, up to 1 month after the treatment. Microbial recovery rate and temporal instability have been associated with several GI conditions. Hence, it is plausible to hypothesise that an already depleted microbiota might suffer with the single dosing method. Severe purging, resembling that induced with the PEG electrolyte solution, does happen during infective gastroenteritis. Therefore, it is possible that the changes we observed may parallel those seen in other forms of diarrhoea. Future studies with larger cohorts from different patient groups would elucidate these points; however, our results do provide an insight into the general effect of bowel cleansing on the intestinal microbiota and specifically the dosing.

Acknowledgements The authors would like to thank the HITChip team: Hans Heilig, Philippe Guilla Puylaert, Ineke Heikamp-de Jong and Wilma Akkermans-van Vliet from University of Wageningen and Laura Huuskonen from University of Helsinki for their excellent technical assistance. This manuscript results in part from collaboration and network activities promoted in the frame of the international network GENIEUR (Genes in irritable bowel syndrome Europe), which is currently funded by the COST program (BM1106, http://www.GENIEUR.eu).

Contributors JJ, AS, LM, RCS, PG, CH and WMdV contributed to the study concept and design. RS, KG and CL collected the clinical samples. JJ and OI contributed to the acquisition of data. JJ, AS, JS, JR and WMdV analysed and interpreted the data. JJ, AS, JS and WMdV drafted the manuscript. RS, AP and WMdW provided administrative, technical or material support. All authors contributed to the critical revision of the manuscript.

Funding This work was partly funded by the Finnish Funding Agency for Technology and Innovation (TEKES) grant 40274/06 and Academy of Finland grants (118602, 1141130, 137389 and 141140) in the context of the Finnish Centre of Excellence in Microbial Food Safety Research (CoE-MiFoSa), as well as by the Graduate School of Applied Biosciences. This study was also supported by an educational grant from Norgine Pharmaceuticals, Norgine House, Harefield, Uxbridge UK to the University of Nottingham and was performed in the NIHR funded Nottingham Digestive Diseases Biomedical Research Unit.

Competing interests RS grant support for sample collection from Norgine; advisory board member of Almirall, Danone and Ironwood; lecturing fees from Abbott and Shire.

Patient consent Obtained.

Ethics approval The study was approved by the National Research Ethics Service (approval 10/H0906/50), the NHS Trust R\&D (approval 10GA018) and the Medicines and Healthcare products Regulatory Agency (MHRA Clinical Trial Authorization CTA 03057/0045/001-0001, protocol 10050).

Provenance and peer review Not commissioned; externally peer reviewed.

Data sharing statement The phylonetic microarray used in the study is a custom made array, hence not MIAME compatible. We have included the raw microarray data from genus level for review purposes.

\section{REFERENCES}

1 Connor A, Tolan D, Hughes $S$, et al. Consensus guidelines for the safe prescription and administration of oral bowel-cleansing agents. Gut 2012;61:1525-32.

2 Strocchi A, Bond JH, Ellis C, et al. Colonic Concentrations of Hydrogen and Methane Following Colonoscopic Preparation with an Oral Lavage Solution. Gastrointest Endosc 1990:36:580-2.

3 Jalanka-Tuovinen J, Salonen A, Nikkilä J, et al. Intestinal microbiota in healthy adults: temporal analysis reveals individual and common core and relation to intestinal symptoms. PLOS ONE 2011;6:e23035.

4 Zoetendal EG, Akkermans AD, De Vos WM. Temperature gradient gel electrophoresis analysis of $16 \mathrm{~S}$ rRNA from human fecal samples reveals stable and host-specific communities of active bacteria. App/ Environ Microbiol 1998;64:3854-9.

5 Rajilic-Stojanovic M, Heilig HG, Tims S, et al. Long-term monitoring of the human intestinal microbiota composition. Environ Microbiol 2012;15:1146-59.

6 Jernberg C, Lofmark S, Edlund C, et al. Long-term impacts of antibiotic exposure on the human intestinal microbiota. Microbiology 2010;156:3216-23.

7 Kamada N, Chen GY, Inohara N, et al. Control of pathogens and pathobionts by the gut microbiota. Nat Immunol 2013;14:685-90.

8 Lahti L, Salojärvi J, Salonen A, et al. Tipping elements in the human intestinal ecosystem. Nat Commun 2014;5:4344.
9 Mai V, Greenwald B, Morris JG Jr, et al. Effect of bowel preparation and colonoscopy on post-procedure intestinal microbiota composition. Gut 2006;55:1822-3.

10 Harrell L, Wang YW, Antonopoulos D, et al. Standard colonic lavage alters the natural state of mucosal-associated microbiota in the human colon. PLOS ONE 2012;7:e32545

11 O'Brien $\mathrm{CL}$, Allison GE, Grimpen $\mathrm{F}$, et al. Impact of colonoscopy bowel preparation on intestinal microbiota. PLOS ONE 2013;8:e62815.

12 Gorkiewicz G, Thallinger GG, Trajanoski $S$, et al. Alterations in the colonic microbiota in response to osmotic diarrhea. PLOS ONE 2013;8:e55817.

13 Johansson ME, Gustafsson JK, Holmen-Larsson J, et al. Bacteria penetrate the normally impenetrable inner colon mucus layer in both murine colitis models and patients with ulcerative colitis. Gut 2014;63:281-91.

14 Tooth D, Garsed K, Singh G, et al. Characterisation of faecal protease activity in irritable bowel syndrome with diarrhoea: origin and effect of gut transit. Gut 2014;63:753-60.

15 Garsed KC, Marciani L, Fields A, et al. Mode of action of a macrogol formulation on distribution of intestinal fluid: a MRI study. Gastroenterology 2012;142:S814.

16 Salonen A, Nikkilä J, Jalanka-Tuovinen J, et al. Comparative analysis of fecal DNA extraction methods with phylogenetic microarray: effective recovery of bacterial and archaeal DNA using mechanical cell lysis. J Microbiol Methods 2010;81:127-34.

17 Rajilic-Stojanovic M, Heilig HG, Molenaar D, et al. Development and application of the human intestinal tract chip, a phylogenetic microarray: analysis of universally conserved phylotypes in the abundant microbiota of young and elderly adults. Environ Microbiol 2009:11:1736-51.

18 Claesson MJ, O'Sullivan 0, Wang Q, et al. Comparative analysis of pyrosequencing and a phylogenetic microarray for exploring microbial community structures in the human distal intestine. PLOS ONE 2009;4:e6669.

19 Lahti L, Torrente A, Elo LL, et al. A fully scalable online pre-processing algorithm for short oligonucleotide microarray atlases. Nucleic Acids Res 2013;41:e110.

20 Nadkarni MA, Martin FE, Jacques NA, et al. Determination of bacterial load by real-time PCR using a broad-range (universal) probe and primers set. Microbiology 2002:148:257-66.

21 Ufnar JA, Wang SY, Christiansen JM, et al. Detection of the nifH gene of Methanobrevibacter smithii: a potential tool to identify sewage pollution in recreational waters. J App/ Microbiol 2006;101:44-52.

22 Heid CA, Stevens J, Livak KJ, et al. Real time quantitative PCR. Genome Res 1996:6:986-94.

23 Roka R, Rosztoczy A, Leveque $M$, et al. A pilot study of fecal serine-protease activity: a pathophysiologic factor in diarrhea-predominant irritable bowel syndrome. Clin Gastroenterol Hepatol 2007;5:550-5.

24 Langille MGI, Zaneveld J, Caporaso JG, et al. Predictive functional profiling of microbial communities using 16S rRNA marker gene sequences. Nat Biotechnol 2013:31:814-21

25 McDonald D, Price MN, Goodrich J, et al. An improved Greengenes taxonomy with explicit ranks for ecological and evolutionary analyses of bacteria and archaea. ISME J 2012:6:610-18.

26 Caporaso JG, Kuczynski J, Stombaugh J, et al. QIIME allows analysis of high-throughput community sequencing data. Nat Methods 2010;7:335-6.

27 Kanehisa M, Goto S. KEGG: kyoto encyclopedia of genes and genomes. Nucleic Acids Res 2000;28:27-30.

28 Huttenhower $C$, Gevers D, Knight $R$, et al. Structure, function and diversity of the healthy human microbiome. Nature 2012;486:207-14.

29 Pop M, Walker AW, Paulson J, et al. Diarrhea in young children from low-income countries leads to large-scale alterations in intestinal microbiota composition. Genome Biol 2014;15:R76.

30 Cohen LB. Split dosing of bowel preparations for colonoscopy: an analysis of its efficacy, safety, and tolerability. Gastrointest Endosc 2010;72:406-12.

31 Nomura T, Ohkusa T, Okayasu I, et al. Mucosa-associated bacteria in ulcerative colitis before and after antibiotic combination therapy. Aliment Pharmacol Ther 2005;21:1017-27

32 Raman M, Ahmed I, Gillevet PM, et al. Fecal microbiome and volatile organic compound metabolome in obese humans with nonalcoholic fatty liver disease. Clin Gastroenterol H 2013;11:868-75.

33 Le Chatelier E, Nielsen T, Qin J, et al. Richness of human gut microbiome correlates with metabolic markers. Nature 2013;500:541-6.

34 Rajilic-Stojanovic M, Biagi E, Heilig HG, et al. Global and deep molecular analysis of microbiota signatures in fecal samples from patients with irritable bowel syndrome. Gastroenterology 2011;141:1792-801.

35 Gecse K, Roka R, Ferrier L, et al. Increased faecal serine protease activity in diarrhoeic IBS patients: a colonic lumenal factor impairing colonic permeability and sensitivity. Gut 2008;57:591-9.

36 Bohe M, Borgstrom A, Genell S, et al. Determination of immunoreactive trypsin, pancreatic elastase and chymotrypsin in extracts of human feces and ileostomy drainage. Digestion 1983;27:8-15.

37 Genell S, Gustafsson BE. Impaired enteric degradation of pancreatic endopeptidases in antibiotic-treated rats. Scand J Gastroenterol 1977;12:801-9.

38 Carroll IM, Ringel-Kulka T, Ferrier L, et al. Fecal protease activity is associated with compositional alterations in the intestinal microbiota. PLOS ONE 2013;8:e78017. 